Article

\title{
Duality for Unified Higher-Order Minimax Fractional Programming with Support Function under Type-I Assumptions
}

\author{
Ramu Dubey ${ }^{1} \mathbb{D}$, Vishnu Narayan Mishra ${ }^{2, *(\mathbb{D})}$, and Rifaqat Ali ${ }^{3}$ \\ 1 Department of Mathematics, J C Bose University of Science and Technology, YMCA, \\ Faridabad 121006, India; rdubeyjiya@gmail.com \\ 2 Department of Mathematics, Indira Gandhi National Tribal University, Lalpur, Amarkantak, Anuppur, \\ Madhya Pradesh 484887, India \\ 3 Department of Mathematics, College of Science and Arts, Muhayil, King Khalid University, \\ 61413 Abha, Saudi Arabia; rifaqat.ali1@gmail.com or rrafat@kku.edu.sa \\ * Correspondence: vishnunarayanmishra@gmail.com
}

Received: 5 September 2019; Accepted: 15 October 2019; Published: 3 November 2019

check for updates

\begin{abstract}
This article is devoted to discussing the nondifferentiable minimax fractional programming problem with type-I functions. We focus our study on a nondifferentiable minimax fractional programming problem and formulate a higher-order dual model. Next, we establish weak, strong, and strict converse duality theorems under generalized higher-order strictly pseudo $(V, \alpha, \rho, d)$-type-I functions. In the final section, we turn our focus to study a nondifferentiable unified minimax fractional programming problem and the results obtained in this paper naturally unify. Further, we extend some previously known results on nondifferentiable minimax fractional programming in the literature.
\end{abstract}

Keywords: duality; support function; nondifferentiable; strictly pseudo $(V, \alpha, \rho, d)$-type-I; unified dual; efficient solutions

\section{Introduction}

Minimax is a decision rule used in decision theory, game theory, statistics, and philosophy for minimizing the possible loss for a worst case (maximum loss) scenario. In general, a minimax problem can be formulated as

$$
\min _{x \in X} \max _{i} f_{i}(x), \quad i=1,2,3, \ldots, m,
$$

where $f_{i}(x)$ is a function defined on the space $X$. Many minimax problems often arise in engineering design, computer-aided-design, circuit design, and optimal control. Some of the problems arising in engineering, economics, and mathematics are of the following form:

Minimize a function $\Theta(x)$ subject to $x \in \Omega$, where $\Theta(x)$ is one of the following functions:

(a) $\Theta(x)=\max _{y \in H} f(x, y)$,

(b) $\Theta(x)=\max _{y \in H(x)} f(x, y)$,

(c) $\Theta(x)=\max _{y \in H_{1}(x)} \min _{z \in H_{2}(x)} f(x, y, z)$,

(d) $\Theta(x)=\max _{y_{1} \in H_{11}(x)} \min _{z_{1} \in H_{21}(x)}, \ldots, \max _{y_{k} \in H_{1 k}(x)} \min _{z_{k} \in H_{2 k}(x)} f\left(x, y_{1}, \ldots, y_{k}, z_{1}, \ldots, z_{k}\right)$, where the sets $H(x), H_{i}(x), H_{i j}$ depend on $x$ and $H, \Omega$ are given sets, 
(e) $\Theta(x)=\max _{i} f_{i}(x), i \in\{1,2,3, \ldots, m\}$.

Such problems often appear in the engineering design theory. In recent years, much attention was paid to the problems described. The minimax theory deals with the following problems:

(1) Necessary and sufficient conditions and their geometric interpretation [1,2];

(2) Steepest-descent directions and their applications to constructing numerical methods. The problems have been widely discussed and studied for the function $(a)$;

(3) Saddle points: The problem of finding saddle points is a special case of minimax problems (see survey [3]);

(4) Optimal control problems with a minimax criterion function.

These facts indicate that minimax theory will continue to be an important tool for solving difficult and interesting problems. In addition, minimax methods provide a paradigm for investigating analogous problems. An exciting future with new unified theories may be expected. Optimization problems, in which both a minimization and a maximization process are performed, are known as minimax problems in the area of mathematical programming. For more details, we refer to Stancu-Minasian [4]. Tanimoto [5] applied these optimality conditions to construct a dual problem and established duality theorems. Many researchers have done work related to the same area [6-14].

Fractional programming is an interesting subject which features in several types of optimization problems, such as inventory problem, game theory, and in many other cases. In addition, it can be used in engineering and economics to minimize a ratio of functions between a given period of time and as a utilized resource in order to measure the efficiency of a system. In these sorts of problems, the objective function is usually given as a ratio of functions in fractional programming from (see $[15,16])$.

Motivated by various concepts of generalized convexity, Liang et al. [17] introduced the concept of $(F, \alpha, \rho, d)$-convex functions. Hachimi and Aghezzaf [18], with prior definitions of generalized convexity, extended the concept further to $(F, \alpha, \rho, d)$-type I functions and gave the sufficient optimality conditions and mixed-type duality results for the multiobjective programming problem.

This paper is divided into four sections. Section 2 contains definitions of higher-order strictly pseudo $(V, \alpha, \rho, d)$-type-I functions. In section 3 , we concentrate our discussion on a nondifferentiable minimax fractional programming problem and formulate the higher-order dual model. We establish duality theorems under higher-order strictly pseudo $(V, \alpha, \rho, d)$-type-I functions. In the final section, we turn our attention to discuss a nondifferentiable mixed-type minimax fractional programming problem and establish duality relations under the same assumptions.

\section{Preliminaries and Definitions}

Throughout this paper, we use $S^{\prime}=\{1,2, \ldots, s\}, M=\{1,2, \ldots, m\}$ and $(z, w, v, \mu, p) \in R^{n} \times R^{n} \times$ $R^{n} \times R_{+}^{m} \times R^{n}$.

Definition 1. Let $Q$ be a compact convex set in $R^{n}$. The support function of $Q$ is denoted by $s(x \mid Q)$ and defined by

$$
s(x \mid Q)=\max \left\{x^{T} y: y \in Q\right\} .
$$

The support function $s(x \mid Q)$, being convex and everywhere finite, has a Clarke subdifferential [8], in the sense of convex analysis. The subdifferential of $s(x \mid Q)$ is given by

$$
\partial s(x \mid Q)=\left\{z \in Q \mid z^{T} x=s(x \mid Q)\right\} .
$$


For any set $S$, the normal case to $S$ at a point $x \in S$, denoted by $N_{S}(x)$ and denoted by

$$
N_{S}(x)=\left\{y \in R^{n}: y^{T}(z-x), \forall z \in S\right\}
$$

It is readily verified that for a compact convex set $Q \in R^{n}, y \in N_{S}(x)$ if and only if $s(x \mid Q)=x^{T} y$ or equivalently, $x$ is in the Clarke subdifferential of s at $y$.

Consider the following nondifferentiable minimax fractional programming problem (FP):

$$
\begin{aligned}
& \text { Minimize } \frac{\xi(x, y)}{\zeta(x, y)}=\sup _{y \in Y} \frac{f(x, y)+s(x \mid C)}{g(x, y)-s(x \mid D)}, \\
& \text { subject to } S=\left\{x \in X: h_{j}(x)+s\left(x \mid E_{j}\right) \leq 0, j \in M\right\},
\end{aligned}
$$

where $Y$ is a compact subject of $R^{m}, f, g: X \times Y \rightarrow R$ and $h_{j}: X \rightarrow R, i \in S^{\prime}$ are continuously differentiable functions on $R^{n} \times R^{m} \cdot f(x, y)+s(x \mid C) \geq 0$ and $g(x, y)-s(x \mid D)>0, \forall x \in S$. C, D, and $E_{j}, j \in M$ are compact convex sets in $R^{m}$, and $s(x \mid C), s(x \mid D)$, and $s\left(x \mid E_{j}\right), j \in M$ designate the support functions of compact sets.

$$
\begin{aligned}
& N(x)=\left\{i \in S^{\prime}: h_{j}(x)=0\right\} \\
& Y(x)=\left\{y \in Y: \frac{f(x, y)+s(x \mid C)}{g(x, y)-s(x \mid D)}=\sup _{z \in Y} \frac{f(x, z)+s(x \mid C)}{g(x, z)-s(x \mid D)}\right\}
\end{aligned}
$$

and

$$
\begin{aligned}
& K(x)=\left\{(s, t, \tilde{y}) \in N \times R_{+}^{s} \times R^{m}: 1 \leq s \leq n+1, t=\left(t_{1}, t_{2}, \ldots, t_{s}\right) \in R_{+}^{s}\right. \\
& \text { with } \left.\sum_{i=1}^{s} t_{i}=1, \bar{y}=\left(\bar{y}_{1}, \bar{y}_{2}, \ldots, \bar{y}_{s}\right) \text { and } \bar{y}_{i} \in Y(x), i \in S\right\} .
\end{aligned}
$$

Assume that $\alpha: X \times X \rightarrow R_{+} \backslash\{0\}, \eta: X \times X \rightarrow R^{n}, \rho \in R$ and $d: X \times X \rightarrow R$ (satisfying $d(x, y)=0 \Leftrightarrow x=y)$. Let $\phi: X \times Y \rightarrow R$ and $\psi_{j}: X \rightarrow R$ be twice differentiable functions.

Definition 2. $\forall j \in M,\left[\phi, \psi_{j}\right]$ is said to be higher-order $(V, \alpha, \rho, d)$-type-I at $\bar{x}$, if $\exists \alpha, \rho, d$, and $\eta$ such that $\forall x \in S, y_{i} \in Y(x)$, and $p \in R^{n}$, we have

$$
\begin{aligned}
\phi\left(x, y_{i}\right)-\phi\left(\bar{x}, y_{i}\right) & -G\left(\bar{x}, y_{i}, p\right)+p^{T} \nabla_{p} G\left(\bar{x}, y_{i}, p\right) \\
\geq & \left\langle\alpha(x, \bar{x})\left\{\nabla \phi\left(\bar{x}, y_{i}\right)+\nabla_{p} G\left(\bar{x}, y_{i}, p\right)\right\}, \eta(x, \bar{x})\right\rangle+\rho_{i} d^{2}(x, \bar{x}), i \in S^{\prime}
\end{aligned}
$$

and

$$
-\psi_{j}(\bar{x})-K_{j}(\bar{x}, p)+p^{T} \nabla_{p} K_{j}(\bar{x}, p) \geq\left\langle\alpha(x, \bar{x})\left\{\nabla \psi_{j}(\bar{x})+\nabla_{p} K_{j}(\bar{x}, p)\right\}, \eta(x, \bar{x})\right\rangle+\rho_{j} d^{2}(x, \bar{x}), j \in M .
$$

Remark 1. In the above definition, if the inequalities appear as strict inequalities, then we say that $\left[\phi, \psi_{j}\right], \forall j \in$ $M$ is higher-order strict $(V, \alpha, \rho, d)$-type- $I$.

Remark 2. If $G\left(\bar{x}, y_{i}, p\right)=\frac{1}{2} p^{T} \nabla^{2} \phi\left(\bar{x}, y_{i}\right) p$ and $\rho_{i}=0, \forall i \in S^{\prime}$, then Definition 2 becomes $\alpha$-type-I at $\bar{x}$ given by [19]. 
Definition 3. $\forall j \in M,\left[\phi, \psi_{j}\right]$ is said to be higher-order pseudoquasi $(V, \alpha, \rho, d)$-type-I at $\bar{x}$, if $\exists \alpha, \rho, d$, and $\eta$ such that $\forall x \in S, y_{i} \in Y(x)$, and $p \in R^{n}$, we have

$$
\begin{aligned}
\phi\left(x, y_{i}\right)-\phi\left(\bar{x}, y_{i}\right)-G\left(\bar{x}, y_{i}, p\right)+p^{T} \nabla_{p} G\left(\bar{x}, y_{i}, p\right)<0 \\
\Rightarrow\left\langle\alpha(x, \bar{x})\left\{\nabla \phi\left(\bar{x}, y_{i}\right)+\nabla_{p} G\left(\bar{x}, y_{i}, p\right)\right\}, \eta(x, \bar{x})\right\rangle+\rho_{i} d^{2}(x, \bar{x})<0, \quad i \in S^{\prime}
\end{aligned}
$$

and

$$
-\psi_{j}(\bar{x})-K_{j}(\bar{x}, p)+p^{T} \nabla_{p} K_{j}(\bar{x}, p) \leq 0 \Rightarrow\left\langle\alpha(x, \bar{x})\left\{\nabla \psi_{j}(\bar{x})+\nabla_{p} K_{j}(\bar{x}, p)\right\}, \eta(x, \bar{x})\right\rangle+\rho_{j} d^{2}(x, \bar{x}) \leq 0, j \in M .
$$

Remark 3. In Definition 3, if $\left\langle\alpha(x, \bar{x})\left\{\nabla \phi\left(\bar{x}, \bar{y}_{i}\right)+p^{T} \nabla_{p} G\left(\bar{x}, \bar{y}_{i}, p\right)\right\}, \eta(x, \bar{x})\right\rangle+\rho_{i} d^{2}(x, \bar{x}) \geq 0$

$$
\Rightarrow \phi\left(x, y_{i}\right)-\phi\left(\bar{x}, y_{i}\right)-G\left(\bar{x}, y_{i}, p\right)+p^{T} \nabla_{p} G\left(\bar{x}, y_{i}, p\right)>0, i \in S^{\prime}
$$

then $\left[\phi, \psi_{j}\right], \forall j \in M$ is higher-order strictly pseudoquasi $(V, \alpha, \rho, d)$-type-I.

Remark 4. If $G\left(\bar{x}, y_{i}, p\right)=\frac{1}{2} p^{T} \nabla^{2} \phi\left(\bar{x}, y_{i}\right) p$ and $\rho_{i}=0, \forall i \in S^{\prime}$, then Definition 3 reduces to $\alpha$-type-I at $\bar{x}$, given by [19].

Theorem 1 (Necessary condition). If $x^{*}$ is an optimal solution of problem (FP) satisfying $<w, x>>0,<$ $v, x>>0$, and $\nabla\left(h_{j}\left(x^{*}\right)+<u_{j}, x^{*}>\right), j \in N\left(x^{*}\right)$ are linearly independent, then $\exists\left(s^{*}, t^{*}, \bar{y}^{*}\right) \in$ $K\left(x^{*}\right), w \in R^{n}, v \in R^{n}$ and $\mu^{*} \in R_{+}^{m}$ such that

$$
\begin{gathered}
\sum_{i=1}^{s^{*}} t_{i}^{*} \nabla\left(\frac{f_{i}\left(x^{*}, \bar{y}_{i}\right)+<w, x^{*}>}{g_{i}\left(x^{*}, \bar{y}_{i}\right)-<v, x^{*}>}\right)+\sum_{j=1}^{m} \mu_{j}^{*} \nabla\left(h_{j}\left(x^{*}\right)+<u_{j}, x^{*}>\right)=0, \\
\sum_{j=1}^{m} \mu_{j}^{*}\left(h_{j}\left(x^{*}\right)+<u_{j}, x^{*}>\right)=0, \\
t_{i}^{*}>0, i \in S^{*}, \sum_{i=1}^{s^{*}} t_{i}^{*}=1, \mu_{j}^{*} \geq 0, j \in M, \\
<w, x^{*}>=s\left(x^{*} \mid C\right), \quad<v, x^{*}>=s\left(x^{*} \mid D\right),<u_{j}, x^{*}>=s\left(x^{*} \mid E_{j}\right) .
\end{gathered}
$$

\section{Higher-Order Nondifferentiable Duality Model}

The study of higher-order duality is more significant due to the computational advantage over second- and first-order duality as it provides tighter bounds due to presence of more parameters. In the present article, we formulate a new type of duality model for a nondifferentiable minimax fractional programming problem and derive duality theorems under generalized convexity assumptions. Additionally, we use the concept of support function as a nondifferentiable term. Consider the following dual (HFD) of the problem (FP):

$$
\begin{aligned}
& \text { (HFD) } \max _{(s, t, \bar{y}) \in K(z)} \sup _{(z, w, v, u, \mu, p) \in H(s, t, \bar{y})} \sum_{i=1}^{s} t_{i}\left[\frac{f\left(z, \bar{y}_{i}\right)+<w, z>}{g\left(z, \bar{y}_{i}\right)-<v, z>}+G\left(z, \bar{y}_{i}, p\right)-p^{T} \nabla_{p} G\left(z, \bar{y}_{i}, p\right)\right] \\
& +\sum_{i=1}^{m} \mu_{j}\left(h_{j}(z)+<u_{j}, z>+K_{j}(z, p)-p^{T} \nabla_{p} K_{j}(z, p)\right) \text {, }
\end{aligned}
$$


where $H(s, t, \bar{y})$ represents the set of all $(z, w, v, u, \mu, p)$ such that

$$
\begin{gathered}
\nabla \sum_{i=1}^{s} t_{i}\left(\frac{f\left(z, \bar{y}_{i}\right)+<w, z>}{g\left(z, \bar{y}_{i}\right)-<v, z>}\right)+\nabla \sum_{j=1}^{m} \mu_{j}\left(h_{j}(z)+<u_{j}, z>\right) \\
+\sum_{i=1}^{s} t_{i} \nabla_{p} G\left(z, \bar{y}_{i}, p\right)+\sum_{j=1}^{m} \mu_{j} \nabla_{p} K_{j}(z, p)=0, \\
t_{i} \geq 0, \sum_{i=1}^{s} t_{i}=1, \mu_{j} \geq 0, i \in S^{\prime}, j \in M, \\
(s, t, \tilde{y}) \in K(z) .
\end{gathered}
$$

Let $T^{0}$ be the feasible set for (HFD).

Theorem 2 (Weak Duality). Let $x \in S$ and $(z, w, v, \mu, s, t, \bar{y}, p) \in T^{0}$. Let

(i) $\left[\frac{f\left(., \bar{y}_{i}\right)+<w, .>}{g\left(., \bar{y}_{i}\right)-<v, .>}, h_{j}()+.<u_{j} .>\right], i \in S^{\prime}, j \in M$ be higher-order $(V, \alpha, \rho, d)$ - type-I at $z$,

(ii) $\sum_{i=1}^{S} t_{i} \rho_{i}+\sum_{j=1}^{m} \mu_{j} \rho_{j} \geq 0$

Then,

$$
\begin{aligned}
& \sup _{y \in Y}\left(\frac{f(x, y)+<w, x>}{g(x, y)-<v, x>}\right) \geq \sum_{i=1}^{s} t_{i} {\left[\left(\frac{f\left(z, \bar{y}_{i}\right)+<w, z>}{g\left(z, \bar{y}_{i}\right)-<v, z>}\right)+G\left(z, \bar{y}_{i}, p\right)-p^{T} \nabla_{p} G\left(z, \bar{y}_{i}, p\right)\right] } \\
&+\sum_{i=1}^{m} \mu_{j}\left(h_{j}(z)+<u_{j}, z>+K_{j}(z, p)-p^{T} \nabla_{p} K_{j}(z, p)\right) .
\end{aligned}
$$

Proof. We shall derive the result by assuming contrary to the above inequality. Suppose

$$
\begin{gathered}
\sup _{y \in Y}\left(\frac{f(x, y)+<w, x>}{g(x, y)-<v, x>}\right)<\sum_{i=1}^{s} t_{i}\left[\left(\frac{f\left(z, \bar{y}_{i}\right)+<w, z>}{g\left(z, \bar{y}_{i}\right)-<v, z>}\right)+G\left(z, \bar{y}_{i}, p\right)-p^{T} \nabla_{p} G\left(z, \bar{y}_{i}, p\right)\right] \\
+\sum_{i=1}^{m} \mu_{j}\left(h_{j}(z)+<u_{j}, z>+K_{j}(z, p)-p^{T} \nabla_{p} K_{j}(z, p)\right) .
\end{gathered}
$$

This implies

$$
\begin{aligned}
& \left(\frac{f\left(x, \bar{y}_{i}\right)+<w, x>}{g\left(x, \bar{y}_{i}\right)-<v, x>}\right)<\sum_{i=1}^{s} t_{i}\left[\left(\frac{f\left(z, \bar{y}_{i}\right)+<w, z>}{g\left(z, \bar{y}_{i}\right)-<v, z>}\right)+G\left(z, \bar{y}_{i}, p\right)-p^{T} \nabla_{p} G\left(z, \bar{y}_{i}, p\right)\right] \\
& +\sum_{i=1}^{m} \mu_{j}\left(h_{j}(z)+<u_{j}, z>+K_{j}(z, p)-p^{T} \nabla_{p} K_{j}(z, p)\right), \text { for all } \bar{y}_{i} \in Y(x), i \in S^{\prime}
\end{aligned}
$$

Further, using $t_{i} \geq 0, i \in S^{\prime}$ and $\sum_{i=1}^{s} t_{i}=1$, we get

$$
\begin{aligned}
\sum_{i=1}^{s} t_{i}\left(\frac{f\left(x, \bar{y}_{i}\right)+<w, x>}{g\left(x, \bar{y}_{i}\right)-<v, x>}\right)< & \sum_{i=1}^{s} t_{i}\left(\frac{f\left(z, \bar{y}_{i}\right)+<w, z>}{g\left(z, \bar{y}_{i}\right)-<v, z>}+G\left(z, \bar{y}_{i}, p\right)-p^{T} \nabla_{p} G\left(z, \bar{y}_{i}, p\right)\right) \\
& +\sum_{i=1}^{m} \mu_{j}\left(h_{j}(z)+<u_{j}, z>+K_{j}(z, p)-p^{T} \nabla_{p} K_{j}(z, p)\right) .
\end{aligned}
$$


By inequality (7), we obtain

$$
\begin{aligned}
\sum_{i=1}^{s} t_{i}\left(\frac{f\left(x, \bar{y}_{i}\right)+<w, x>}{g\left(x, \bar{y}_{i}\right)-<v, x>}\right)<\sum_{i=1}^{s} t_{i}( & \left.\frac{f\left(z, \bar{y}_{i}\right)+<w, z>}{g\left(z, \bar{y}_{i}\right)-<v, z>}+G\left(z, \bar{y}_{i}, p\right)-p^{T} \nabla_{p} G\left(z, \bar{y}_{i}, p\right)\right) \\
& +\sum_{i=1}^{m} \mu_{j}\left(h_{j}(z)+<u_{j}, z>+K_{j}(z, p)-p^{T} \nabla_{p} K_{j}(z, p)\right) .
\end{aligned}
$$

By hypothesis $(i)$, we get

$$
\begin{aligned}
& \left(\frac{f\left(x, \bar{y}_{i}\right)+<w, x>}{g\left(x, \bar{y}_{i}\right)-<v, x>}\right)-\left(\frac{f\left(z, \bar{y}_{i}\right)+<w, z>}{g\left(z, \bar{y}_{i}\right)-<v, z>}\right)-G\left(z, \bar{y}_{i}, p\right)+p^{T} \nabla_{p} G\left(z, \bar{y}_{i}, p\right) \\
& \quad \geq\left\langle\alpha(x, z)\left\{\nabla\left(\frac{f\left(z, \bar{y}_{i}\right)+<w, z>}{g\left(z, \bar{y}_{i}\right)-<v, z>}\right)+\nabla_{p} G\left(z, \bar{y}_{i}, p\right)\right\}, \eta(x, z)\right\rangle+\rho_{i} d^{2}(x, z), i \in S^{\prime}
\end{aligned}
$$

and

$$
\begin{aligned}
-h_{j}(z)+< & u_{j}, z>-K_{j}(z, p)+p^{T} \nabla_{p} K_{j}(z, p) \\
& \geq\left\langle\alpha(x, z)\left\{\nabla\left(h_{j}(z)+<u_{j}, z>\right)+\nabla_{p} K_{j}(z, p)\right\}, \eta(x, z)\right\rangle+\rho_{j} d^{2}(x, z), j \in M .
\end{aligned}
$$

Multiplying the first inequality by $t_{i} \geq 0, i \in S^{\prime}$ and the second by $\mu_{j} \geq 0, j \in M$ with $\sum_{i=1}^{s} t_{i}=1$, we get

$$
\begin{aligned}
& \sum_{i=1}^{s} t_{i}\left[\left(\frac{f\left(x, \bar{y}_{i}\right)+<w, x>}{g\left(x, \bar{y}_{i}\right)-<v, x>}\right)-\left(\frac{f\left(z, \bar{y}_{i}\right)+<w, z>}{g\left(z, \bar{y}_{i}\right)-<v, z>}\right)-G\left(z, \bar{y}_{i}, p\right)+p^{T} \nabla_{p} G\left(z, \bar{y}_{i}, p\right)\right] \\
& \quad \geq\left\langle\alpha(x, z)\left\{\nabla \sum_{i=1}^{s} t_{i}\left(\frac{f\left(z, \bar{y}_{i}\right)+<w, z>}{g\left(z, \bar{y}_{i}\right)-<v, z>}\right)+\nabla_{p} G\left(z, \bar{y}_{i}, p\right)\right\}, \eta(x, z)\right\rangle+\sum_{i=1}^{s} t_{i} \rho_{i} d^{2}(x, z)
\end{aligned}
$$

and

$$
\begin{aligned}
-\sum_{j=1}^{m} \mu_{j}\left(h_{j}(z)+<u_{j}, z>-K_{j}(z, p)+p^{T} \nabla_{p} K_{j}(z, p)\right) \\
\quad \geq\left\langle\alpha(x, z)\left\{\nabla \sum_{j=1}^{m} \mu_{j}\left(h_{j}(z)+<u_{j}, z>\right)+\nabla_{p} K_{j}(z, p)\right\}, \eta(x, z)\right\rangle+\sum_{j=1}^{m} \mu_{j} \rho_{j} d^{2}(x, z) .
\end{aligned}
$$

The above inequalities yield

$$
\begin{gathered}
\sum_{i=1}^{s} t_{i}\left[\left(\frac{f\left(x, \bar{y}_{i}\right)+<w, x>}{g\left(x, \bar{y}_{i}\right)-<v, x>}\right)-\left(\frac{f\left(z, \bar{y}_{i}\right)+<w, z>}{g\left(z, \bar{y}_{i}\right)-<v, z>}\right)\right]-\sum_{j=1}^{m} \mu_{j}\left(h_{j}(z)+<u_{j}, z>\right) \\
-\left\{\sum_{i=1}^{s} t_{i}\left(G\left(z, \bar{y}_{i}, p\right)-p^{T} \nabla_{p} G\left(z, \bar{y}_{i}, p\right)\right)-\sum_{j=1}^{m} \mu_{j}\left(K_{j}(z, p)-p^{T} \nabla_{p} K_{j}(z, p)\right)\right\} \\
\geq\left\langle\alpha ( x , z ) \left\{\sum_{i=1}^{s} t_{i} \nabla\left(\frac{f\left(z, \bar{y}_{i}\right)+<w, z>}{g\left(z, \bar{y}_{i}\right)-<v, z>}\right)+\sum_{j=1}^{m} \mu_{j}\left(\nabla h_{j}(z)+<u_{j}, z>\right)+\sum_{i=1}^{s} t_{i} \nabla_{p} G\left(z, \bar{y}_{i}, p\right)\right.\right. \\
\left.\left.+\sum_{j=1}^{m} \mu_{j} \nabla_{p} K_{j}(z, p)\right\}, \eta(x, z)\right\rangle+\left(\sum_{i=1}^{s} t_{i} \rho_{i}+\sum_{j=1}^{m} \mu_{j} \rho_{j}\right) d^{2}(x, z) .
\end{gathered}
$$


The above inequality together with (11), $\alpha(x, z)>0$, and hypothesis (ii) yield

$$
\begin{aligned}
\left\langle\sum_{i=1}^{s} t_{i} \nabla\left(\frac{f\left(z, \bar{y}_{i}\right)+<w, z>}{g\left(z, \bar{y}_{i}\right)-<v, z>}\right)\right. & +\sum_{j=1}^{m} \mu_{j} \nabla\left(h_{j}(z)+<u_{j}, z>\right) \\
& \left.+\sum_{i=1}^{s} t_{i} \nabla_{p} G\left(z, \bar{y}_{i}, p\right)+\sum_{j=1}^{m} \mu_{j} \nabla_{p} K_{j}(z, p), \eta(x, z)\right\rangle<0,
\end{aligned}
$$

which contradicts (5). This completes the proof.

Theorem 3 (Strong duality). Suppose the set $\left\{\nabla\left(h_{j}\left(x^{*}\right)+<u_{j}, x^{*}>\right)\right\}_{j \in N\left(x^{*}\right)}$ is linearly independent. Let an optimal solution of (FP) be $x^{*}$, further, suppose

$$
\begin{gathered}
G\left(x^{*}, \bar{y}_{i}^{*}, 0\right)=\nabla_{p^{*}} G\left(x^{*}, \bar{y}_{i}^{*}, 0\right)=0, i \in S^{*}, \\
K_{j}\left(x^{*}, 0\right)=\nabla_{p^{*}} K_{j}\left(x^{*}, 0\right)=0, j \in M .
\end{gathered}
$$

Then, there exist $\left(s^{*}, t^{*}, \bar{y}^{*}\right) \in K\left(x^{*}\right)$ and $\left(x^{*}, w^{*}, v^{*}, u^{*}, v^{*}, s^{*}, t^{*}, \bar{y}^{*}, p^{*}\right) \in H\left(s^{*}, t^{*}, \bar{y}^{*}\right)$ such that $\left(x^{*}, w^{*}, v^{*}, \mu^{*}, s^{*}, t^{*}, \bar{y}^{*}, p^{*}=0\right) \in T^{0}$ and the objectives have the equal values. Moreover, if all the conditions of Weak duality theorem hold for any $(z, w, v, \mu, s, t, \bar{y}, p) \in T^{0}$, then $\left(x^{*}, w^{*}, v^{*}, u^{*}, \mu^{*}, s^{*}, t^{*}, \bar{y}^{*}, p^{*}=0\right)$ is an optimal solution of (HFD).

Proof. By Theorem $1, \exists\left(s^{*}, t^{*}, \bar{y}^{*}\right) \in K\left(x^{*}\right)$ such that

$$
\begin{gathered}
\sum_{i=1}^{s^{*}} t_{i}^{*} \nabla\left(\frac{f\left(x^{*}, \bar{y}_{i}\right)+<w, x^{*}>}{g\left(x^{*}, \bar{y}_{i}\right)-<v, x^{*}>}\right)+\sum_{j=1}^{m} \mu_{j}^{*} \nabla\left(h_{j}\left(x^{*}\right)+<u_{j}, x^{*}>\right)=0, \\
\sum_{j=1}^{m} \mu_{j}^{*}\left(h_{j}\left(x^{*}\right)+<u_{j}, x^{*}>\right)=0, \\
t_{i}^{*} \geq 0, i \in S^{\prime}, \sum_{i=1}^{s} t_{i}^{*}=1, \mu_{j}^{*} \geq 0, j \in M, \\
<w, x^{*}>=s\left(x^{*} \mid C\right), \quad<v, x^{*}>=s\left(x^{*} \mid D\right), \quad<u_{j}, x^{*}>=s\left(x^{*} \mid E_{j}\right), j \in M,
\end{gathered}
$$

which, from (17) and (18), imply $\left(x^{*}, w^{*}, v^{*}, \mu^{*}, s^{*}, t^{*}, \bar{y}^{*}, p^{*}=0\right) \in T^{0}$ and the problems (FP) and (HFD) have the same objective value. The point $\left(x^{*}, w^{*}, v^{*}, \mu^{*}, s^{*}, t^{*}, \bar{y}^{*}, p^{*}=0\right)$ is an optimal solution for (HFD) follows from Theorem 2 . This completes the proof.

Theorem 4 (Strict converse duality). Suppose that $x^{*}$ and $\left(z^{*}, w^{*}, v^{*}, v^{*}, s^{*}, t^{*}, \bar{y}^{*}, p^{*}\right)$ are the optimal solutions of (FP) and (HFD), respectively. Let

(i) $\left[\frac{f\left(., \bar{y}_{i}^{*}\right)+<w^{*},>}{g\left(., \bar{y}_{i}^{*}\right)-<v^{*}, .>}, h_{j}()+.<u_{j}^{*}>\right], i \in S^{\prime}, j \in M$ be higher-order strictly $(V, \alpha, \rho, d)$ - type-I and the set $\left\{\nabla h_{j}\left(x^{*}\right)+<u_{j}^{*}, .>, j \in N\left(x^{*}\right)\right\}$ be linearly independent,

(ii) $\sum_{i=1}^{s^{*}} t_{i}^{*} \rho_{i}^{*}+\sum_{j=1}^{m} \mu_{j}^{*} \rho_{j}^{*} \geq 0$.

Then, $z^{*}=x^{*}$. 
Proof. Suppose contrary to the result that $z^{*} \neq x^{*}$. From Theorem 3, we have

$$
\begin{array}{r}
\sup _{y \in Y}\left(\frac{f\left(x^{*}, y^{*}\right)+<w^{*}, x^{*}>}{g\left(x^{*}, y^{*}\right)-<v^{*}, x^{*}>}\right) \leq \sum_{i=1}^{s^{*}} t_{i}^{*}\left(\frac{f\left(z^{*}, \bar{y}_{i}^{*}\right)+<w^{*}, z^{*}>}{g\left(z^{*}, \bar{y}_{i}^{*}\right)-<v^{*}, z^{*}>}\right)+\sum_{i=1}^{m} \mu_{j}^{*}\left(h_{j}\left(z^{*}\right)+<u_{j}^{*}, z^{*}>\right. \\
\quad-\sum_{i=1}^{s^{*}} t_{i}^{*}\left(G\left(z^{*}, \bar{y}_{i}^{*}, p^{*}\right)-p^{* T} \nabla_{p^{*}} G\left(z^{*}, \bar{y}_{i}^{*}, p^{*}\right)\right)+\sum_{i=1}^{m} \mu_{j}^{*}\left(K_{j}\left(z^{*}, p^{*}\right)-p^{* T} \nabla_{p^{*}} K_{j}\left(z^{*}, p^{*}\right)\right) .
\end{array}
$$

Thus, we obtain

$$
\begin{gathered}
\left(\frac{f\left(x^{*}, y_{i}^{*}\right)+<w^{*}, x^{*}>}{g\left(x^{*}, y_{i}^{*}\right)-<v^{*}, x^{*}>}\right) \leq \sum_{i=1}^{s^{*}} t_{i}^{*}\left(\frac{f\left(z^{*}, \bar{y}_{i}^{*}\right)+<w^{*}, z^{*}>}{g\left(z^{*}, \bar{y}_{i}^{*}\right)-<v^{*}, z^{*}>}\right)+\sum_{i=1}^{m} \mu_{j}^{*}\left(h_{j}\left(z^{*}\right)+<u_{j}^{*}, z^{*}>\right) \\
-\sum_{i=1}^{s^{*}} t_{i}^{*}\left(G\left(z^{*}, \bar{y}_{i}^{*}, p^{*}\right)-p^{* T} \nabla_{p^{*}} G\left(z^{*}, \bar{y}_{i}^{*}, p^{*}\right)\right)+\sum_{i=1}^{m} \mu_{j}^{*}\left(K_{j}\left(z^{*}, \bar{y}_{i}^{*}, p^{*}\right)\right. \\
\left.-p^{* T} \nabla_{p^{*}} K_{j}\left(z^{*}, \bar{y}_{i}^{*}, p^{*}\right)\right), \text { for all } \bar{y}_{i}^{*} \in Y\left(x^{*}\right), i \in S^{\prime *} .
\end{gathered}
$$

Following on the lines of Theorem 2, we get

$$
\begin{gathered}
\sum_{i=1}^{s^{*}} t_{i}^{*}\left(\frac{f\left(x^{*}, y_{i}^{*}\right)+<w^{*}, x^{*}>}{g\left(x^{*}, y_{i}^{*}\right)-<v^{*}, x^{*}>}\right) \leq \sum_{i=1}^{s^{*}} t_{i}^{*}\left(\frac{f\left(z^{*}, \bar{y}_{i}^{*}\right)+<w^{*}, z^{*}>}{g\left(z^{*}, \bar{y}_{i}^{*}\right)-<v^{*}, z^{*}>}\right)+\sum_{i=1}^{m} \mu_{j}^{*}\left(h_{j}\left(z^{*}\right)+<u_{j}^{*}, z^{*}>\right) \\
-\sum_{i=1}^{s^{*}} t_{i}^{*}\left(G\left(z^{*}, \bar{y}_{i}^{*}, p^{*}\right)-p^{* T} \nabla_{p^{*}} G\left(z^{*}, \bar{y}_{i}^{*}, p^{*}\right)\right)+\sum_{i=1}^{m} \mu_{j}^{*}\left(K_{j}\left(z^{*}, p^{*}\right)-p^{* T} \nabla_{p^{*}} K_{j}\left(z^{*}, p^{*}\right)\right) .
\end{gathered}
$$

From hypothesis $(i)$, we have

$$
\begin{aligned}
& \left(\frac{f\left(x^{*}, \bar{y}_{i}^{*}\right)+<w^{*}, x^{*}>}{g\left(x^{*}, \bar{y}_{i}\right)^{*}-<v^{*}, x^{*}>}\right)-\left(\frac{f\left(z^{*}, \bar{y}_{i}^{*}\right)+<w^{*}, z^{*}>}{g\left(z^{*}, \bar{y}_{i}^{*}\right)-<v^{*}, z^{*}>}\right)-G\left(z^{*}, \bar{y}_{i}^{*}, p^{*}\right)+p^{* T} \nabla_{p^{*}} G\left(z^{*}, \bar{y}_{i}^{*}, p^{*}\right) \\
& >\left\langle\alpha\left(x^{*}, z^{*}\right)\left\{\nabla\left(\frac{f\left(z^{*}, \bar{y}_{i}^{*}\right)+<w^{*}, z^{*}>}{g\left(z^{*}, \bar{y}_{i}^{*}\right)-<v^{*}, z^{*}>}\right)+\nabla_{p^{*}} G\left(z^{*}, \bar{y}_{i}^{*}, p^{*}\right)\right\}, \eta\left(x^{*}, z^{*}\right)\right\rangle+\rho_{i}^{*} d^{2}\left(x^{*}, z^{*}\right), i \in S^{*}
\end{aligned}
$$

and

$$
\begin{aligned}
& -\left(h_{j}\left(z^{*}\right)+<u_{j}^{*}, z^{*}>\right)-K_{j}\left(z^{*}, p^{*}\right)+p^{* T} \nabla_{p^{*}} K_{j}\left(z^{*}, p^{*}\right) \\
& \quad>\left\langle\alpha\left(x^{*}, z^{*}\right)\left\{\nabla\left(h_{j}\left(z^{*}\right)+<u_{j}^{*}, z^{*}>\right)+\nabla_{p^{*}} K_{j}\left(z^{*}, p^{*}\right)\right\}, \eta\left(x^{*}, z^{*}\right)\right\rangle+\rho_{j}^{*} d^{2}\left(x^{*}, z^{*}\right), j \in M .
\end{aligned}
$$

Multiplying the first inequality by $t_{i}^{*} \geq 0, i \in S^{\prime}$ and the second by $\mu_{j}^{*} \geq 0, j=1 \in M$ with $\sum_{i=1}^{s^{*}} t_{i}^{*}=1$, we get

$$
\begin{array}{r}
\sum_{i=1}^{s^{*}} t_{i}^{*}\left[\frac{f\left(x^{*}, \bar{y}_{i}^{*}\right)+<w^{*}, x^{*}>}{g\left(x^{*}, \bar{y}_{i}^{*}\right)-<v^{*}, x^{*}>}-\frac{f\left(z^{*}, \bar{y}_{i}^{*}\right)+<w^{*}, z^{*}>}{g\left(z^{*}, \bar{y}_{i}^{*}\right)-<v^{*}, z^{*}>}\right]-\sum_{i=1}^{s^{*}} t_{i}^{*}\left(G\left(z^{*}, \bar{y}_{i}^{*}, p^{*}\right)\right. \\
\left.+p^{* T} \nabla_{p^{*}} G\left(z^{*}, \bar{y}_{i}^{*}, p^{*}\right)\right)>\left\langle\alpha ( x ^ { * } , z ^ { * } ) \left\{\sum_{i=1}^{s^{*}} t_{i}^{*} \nabla\left(\frac{f\left(z^{*}, \bar{y}_{i}^{*}\right)+<w^{*}, x^{*}>}{g\left(z^{*}, \bar{y}_{i}^{*}\right)-<v^{*}, z^{*}>}\right)\right.\right. \\
\left.\left.+\sum_{i=1}^{s^{*}} t_{i}^{*} \nabla_{p^{*}} G\left(z^{*}, \bar{y}_{i}^{*}, p^{*}\right)\right\}, \eta\left(x^{*}, z^{*}\right)\right\rangle+\sum_{i=1}^{s^{*}} t_{i}^{*} \rho_{i} d^{2}\left(x^{*}, z^{*}\right)
\end{array}
$$


and

$$
\begin{aligned}
& -\sum_{j=1}^{m} \mu_{j}^{*}\left(h_{j}\left(z^{*}\right)+<u_{j}^{*}, z^{*}>-K_{j}\left(x^{*}, p^{*}\right)+p^{* T} \nabla_{p^{*}} K_{j}\left(z^{*}, p^{*}\right)\right. \\
& \quad>\left\langle\alpha\left(x^{*}, z^{*}\right)\left\{\sum_{j=1}^{m} \mu_{j}^{*} \nabla\left(h_{j}\left(z^{*}\right)+<u_{j}^{*}, z^{*}>\right)+\sum_{j=1}^{m} \mu_{j}^{*} \nabla_{p^{*}} K_{j}\left(x^{*}, p^{*}\right)\right\}, \eta\left(x^{*}, z^{*}\right)\right\rangle+\sum_{j=1}^{m} \mu_{j}^{*} \rho_{j} d^{2}\left(x^{*}, z^{*}\right) .
\end{aligned}
$$

The above inequalities yield

$$
\begin{aligned}
& \sum_{i=1}^{s^{*}} t_{i}^{*}\left[\left(\frac{f\left(x^{*}, \bar{y}_{i}^{*}\right)+<w^{*}, x^{*}>}{g\left(x^{*}, \bar{y}_{i}^{*}\right)-<v^{*}, x^{*}>}\right)-\left(\frac{f\left(z^{*}, \bar{y}_{i}^{*}\right)+<w^{*}, z^{*}>}{g\left(z^{*}, \bar{y}_{i}^{*}\right)-<v^{*}, z^{*}>}\right)\right]-\sum_{j=1}^{m} \mu_{j}^{*}\left(h_{j}\left(z^{*}\right)+<u_{j}^{*}, z^{*}>\right) \\
& -\sum_{j=m}^{m} \mu_{j}^{*}\left(K_{j}\left(x^{*}, p^{*}\right)-p^{* T} \nabla_{p^{*}} K_{j}\left(x^{*}, p^{*}\right)\right)-\sum_{i=1}^{s^{*}} t_{i}^{*}\left(G\left(z^{*}, \bar{y}_{i}^{*}, p^{*}\right)-p^{* T} \nabla_{p^{*}} G\left(z^{*}, \bar{y}_{i}^{*}, p^{*}\right)\right)- \\
& \sum_{j=1}^{m} \mu_{j}^{*}\left(h_{j}\left(z^{*}\right)+<u_{j}^{*}, z^{*}>-K_{j}\left(z^{*}, p^{*}\right)+p^{* T} \nabla_{p^{*}} K_{j}\left(z^{*}, p^{*}\right)\right) \\
& >\left\langle\alpha ( x ^ { * } , z ^ { * } ) \left[\sum_{i=1}^{s^{*}} t_{i}^{*} \nabla\left(\frac{f\left(z^{*}, \bar{y}_{i}^{*}\right)+<w^{*}, z^{*}>}{g\left(z^{*}, \bar{y}_{i}^{*}\right)-<v^{*}, z^{*}>}\right)+\sum_{j=1}^{m} \mu_{j}^{*}\left(\nabla h_{j}\left(z^{*}\right)+<u_{j}^{*}, z^{*}>\right)\right.\right. \\
& \left.\left.\quad+\left\{\sum_{i=1}^{s^{*}} t_{i}^{*} \nabla_{p^{*}} G\left(z^{*}, \bar{y}_{i}^{*}, p^{*}\right)+\sum_{j=1}^{m} \mu_{j}^{*} \nabla_{p^{*}} K_{j}\left(z^{*}, p^{*}\right)\right\}\right], \eta\left(x^{*}, z^{*}\right)\right\rangle+\left(\sum_{i=1}^{s} t_{i}^{*} \rho_{i}^{*}+\sum_{j=1}^{m} \mu_{j}^{*} \rho_{j}^{*}\right) d^{2}\left(x^{*}, z^{*}\right) .
\end{aligned}
$$

It follows from (11), $\alpha\left(x^{*}, z^{*}\right)>0$, and hypothesis (ii) that

$$
\begin{aligned}
& \sum_{i=1}^{s^{*}} t_{i}^{*}\left(\frac{f\left(x^{*}, \bar{y}_{i}^{*}\right)+<w^{*}, x^{*}>}{g\left(x^{*}, \bar{y}_{i}^{*}\right)-<v^{*}, x^{*}>}\right)>\sum_{i=1}^{s^{*}} t_{i}^{*}\left(\frac{f\left(z^{*}, \bar{y}_{i}^{*}\right)+<w^{*}, z^{*}>}{g\left(z^{*}, \bar{y}_{i}^{*}\right)-<v^{*}, z^{*}>}\right)+\sum_{j=1}^{m} \mu_{j}^{*}\left(h_{j}\left(z^{*}\right)+<u_{j}^{*}, z^{*}>\right) \\
& -\sum_{i=1}^{s^{*}} t_{i}^{*}\left(G\left(z^{*}, \bar{y}_{i}^{*}, p^{*}\right)+p^{* T} \nabla_{p^{*}} G\left(z^{*}, \bar{y}_{i}^{*}, p^{*}\right)\right)-\sum_{j=1}^{m} \mu_{j}^{*}\left(K_{j}\left(z^{*}, p^{*}\right)+p^{* T} \nabla_{p^{*}} K_{j}\left(z^{*}, p^{*}\right)\right),
\end{aligned}
$$

which contradicts (25). Hence, $z^{*}=x^{*}$.

\section{Mixed-Type Higher-Order Duality Model}

Consider the following higher-order unified dual (HMFD) to (FP):

$$
\text { (HMFD) } \begin{aligned}
\max _{(s, t, \bar{y}) \in K(z)} & \sup _{(z, w, v, u, p) \in H(s, t, \bar{y})} \sum_{i=1}^{s} t_{i}\left(\frac{f\left(z, \bar{y}_{i}\right)+<w, z>}{g\left(z, \bar{y}_{i}\right)-<v, z>}\right)+\sum_{j \in J_{0}} \mu_{j}\left(h_{j}(z)+<u_{j}, z>\right) \\
& +\sum_{i=1}^{s} t_{i}\left(G\left(z, \bar{y}_{i}, p\right)-p^{T} \nabla_{p} G\left(z, \bar{y}_{i}, p\right)\right)+\sum_{j \in J_{0}} \mu_{j}\left(K_{j}(z, p)-p^{T} \nabla_{p} K_{j}(z, p)\right),
\end{aligned}
$$

where $H(s, t, \bar{y})$ represents the set of all $(z, w, v, \mu, p)$ such that

$$
\begin{aligned}
& \nabla \sum_{i=1}^{s} t_{i}\left(\frac{f\left(z, \bar{y}_{i}\right)+<w, z>}{g\left(z, \bar{y}_{i}\right)-<v, z>}\right)+\nabla \sum_{j=1}^{m} \mu_{j}\left(h_{j}(z)+<u_{j}, z>\right) \\
&+\sum_{i=1}^{s} t_{i} \nabla_{p} G\left(z, \bar{y}_{i}, p\right)+\sum_{j=1}^{m} \mu_{j} \nabla_{p} K_{j}(z, p)=0, \\
& \sum_{j \in J_{\beta}} \mu_{j}\left(h_{j}(z)+<u_{j}, z>\right)+\sum_{j \in J_{\beta}} \mu_{j}\left(K_{j}(z, p)-p^{T} \nabla_{p} K_{j}(z, p)\right) \geq 0, \beta=1,2, \ldots, r,
\end{aligned}
$$




$$
t_{i} \geq 0, \sum_{i=1}^{s} t_{i}=1, \mu_{j} \geq 0, i \in S^{\prime}, j \in M
$$

where $J_{\beta} \subseteq M, \beta=0,1,2, \ldots, r$ with $\bigcup_{\beta=0}^{r} J_{\beta}=M$ and $J_{\beta} \cap J_{\gamma}=\phi$, if $\beta \neq \gamma$. Let $W^{0}$ be the feasible set for (HMFD).

Theorem 5 (Weak duality). Let $x \in S$ and $(z, w, v, \mu, s, t, \bar{y}, p) \in W^{0}$. Let

(i) $\left[\sum_{i=1}^{s} t_{i}\left(\frac{f\left(., \bar{y}_{i}\right)+<w_{,} .>}{g\left(., \bar{y}_{i}\right)-<v, .>}\right)+\sum_{j \in J_{0}} \mu_{j}\left(h_{j}()+.<u_{j}, .>\right), \sum_{j \in J_{\beta}} \mu_{j}\left(h_{j}()+.<u_{j}, .>\right)\right], \beta=1,2, \ldots, r b e$ higher-order pseudoquasi $(V, \alpha, \rho, d)$-type $-I$,

(ii) $\sum_{i=1}^{s} t_{i} \rho_{i}+\sum_{j=1}^{m} \mu_{j} \rho_{j} \geq 0$

Then,

$$
\begin{aligned}
\sup _{y \in Y}\left(\frac{f(x, y)+<w, x>}{g(x, y)-<v, x>}\right) & \geq \sum_{i=1}^{s} t_{i}\left(\frac{f\left(z, \bar{y}_{i}\right)+<w, z>w}{g\left(z, \bar{y}_{i}\right)-<v, x>}\right)+\sum_{j \in J_{0}} \mu_{j}\left(h_{j}(z)+<u_{j}, z>\right) \\
& +\sum_{i=1}^{s} t_{i}\left(G\left(z, \bar{y}_{i}, p\right)-p^{T} \nabla_{p} G\left(z, \bar{y}_{i}, p\right)+\sum_{j \in J_{0}} \mu_{j}\left(K_{j}(z, p)-p^{T} \nabla_{p} K_{j}(z, p)\right) .\right.
\end{aligned}
$$

Proof. Proof follows on the lines of Theorem 2.

Theorem 6 (Strong duality). Suppose the set $\left\{\nabla\left(h_{j}\left(x^{*}\right)+<u_{j}, x^{*}>\right)\right\}_{j \in N\left(x^{*}\right)}$ is linearly independent. Let an optimal solution of (FP) be $x^{*}$, further, suppose

$$
\begin{gathered}
G\left(x^{*}, \bar{y}_{i}^{*}, 0\right)=\nabla_{p^{*}} G\left(x^{*}, \bar{y}_{i}^{*}, 0\right)=0, i \in S^{\prime *}, \\
K_{j}\left(x^{*}, 0\right)=\nabla_{p^{*}} K_{j}\left(x^{*}, 0\right)=0, j \in M .
\end{gathered}
$$

Then, $\exists\left(s^{*}, t^{*}, \bar{y}^{*}\right) \in K\left(x^{*}\right)$ and $\left(x^{*}, w^{*}, v^{*}, u^{*}, v^{*}, s^{*}, t^{*}, \bar{y}^{*}, p^{*}\right) \in H\left(s^{*}, t^{*}, \bar{y}^{*}\right)$ such that $\left(x^{*}, w^{*}, v^{*}, \mu^{*}, s^{*}, t^{*}, \bar{y}^{*}, p^{*}=0\right) \in W^{0}$ and the two objectives have the equal values. In addition, if all the conditions of Weak duality theorem hold for any $\left(z, w, v, \mu, s, t, u^{*}, \bar{y}, p\right) \in W^{0}$, then $\left(x^{*}, w^{*}, v^{*}, \mu^{*}, u^{*}, s^{*}, t^{*}, \bar{y}^{*}, p^{*}=0\right)$ is an optimal solution of (HMFD).

Proof. The proof can be obtained following the lines of Theorem 3.

Theorem 7 (Strict converse duality). Let $x^{*}$ and $\left(z^{*}, w^{*}, v^{*}, v^{*}, s^{*}, u^{*}, t^{*}, \bar{y}^{*}, p^{*}\right)$ be the optimal solutions of $(F P)$ and (HMFD), respectively. Let

(i) $\left[\frac{f\left(., \bar{y}_{i}^{*}\right)+<w^{*},>}{g\left(., \bar{y}_{i}^{*}\right)-<v^{*},>}+\sum_{j \in J_{0}} \mu_{j}^{*}\left[h_{j}+<u_{j}^{*},>\right], \sum_{j \in J_{\beta}} \mu_{j}^{*}\left[h_{j}+<u_{j}^{*},>\right]\right], \beta=1,2, \ldots, r$ be higher-order strictly pseudo $(V, \alpha, \rho, d)$ - type-I and $\nabla\left(h_{j}\left(x^{*}\right)+<u_{j}^{*}, x^{*}>\right), j \in N\left(x^{*}\right)$ be linearly independent,

(ii) $\sum_{i=1}^{s^{*}} t_{i}^{*} \rho_{i}+\sum_{j=1}^{m} \mu_{j}^{*} \rho_{j} \geq 0$.

Then, $z^{*}=x^{*}$.

Proof. The proof can be derived following the steps of Theorem 4 . 


\section{Conclusions}

In this paper, we discussed higher-order duality theorems for two types of dual models of nondifferentiable minimax fractional programming problems under strictly pseudo $(V, \alpha, \rho, d)$-type-I functions. The question arises as to whether the second/higher-order duality theorems developed in this paper hold for the complex minimax fractional programming problem. This will orient the future task of the authors.

Author Contributions: Formal Analysis: V.N.M., Editing: R.A. and Investigation: R.D. All authors contributed equally to this research. The research was carried out by all authors. The manuscript was prepared together and they all read and approved the final version.

Funding: The authors extend their appreciation to the "Deanship of Scientific Research" at King Khalid University for funding this work through research groups program under grant number R.G.P.1/152/40.

Acknowledgments: The authors are thankful to the anonymous referees and editor for their valuable suggestions, which have substantially improved the presentation of the paper.

Conflicts of Interest: The authors declare no conflict of interest.

\section{References}

1. Beresnev, V.V. Necessary conditions for extremum in a convex maximin problem on connected sets. Kibernetika 1972, 2, 87-91.

2. Demyanov, V.F. Minimax, Directional Differentiability; Leningrad Univ. Press: Leningrad, Russia, 1974.

3. Demyanov, V.F.; Pevnyi, A.B. Numerical methods for finding saddle points. USSR Comput. Math. Math. Phys. 1972, 12, 11-52. [CrossRef]

4. Stancu-Minasian, I.M. Fractional Programming, Theory, Methods and Applications; Kluwer Academic Publishers: Dordrecht, The Netherlands, 1997.

5. Tanimoto, S. Duality for a class of nondifferentiable mathematical programming problems. J. Math. Anal. Appl. 1981, 7, 283-294. [CrossRef]

6. Liang, Z.A.; Shi, Z.W. Optimality conditions and duality for a minimax fractional programming with generalized convexity. J. Math. Anal. Appl. 2003, 277, 474-488. [CrossRef]

7. Lai, H.C.; Lee, J.C. On duality theorems for a nondifferentiable minimax fractional programming. J. Comput. Appl. Math. 2002, 146, 115-126. [CrossRef]

8. Yuan, D.H.; Liu, X.L.; Chinchuluun, A.; Pardalos, P.M. Nondifferentiable minimax fractional programming problems. J. Optim. Theory Appl. 2006, 129, 185-199. [CrossRef]

9. Mishra, S.K.; Lai, K.K.; Singh, V. Optimality and duality for minimax fractional programming with support function under $(C, \alpha, \rho, d)$-convexity. J. Comput. Appl. Math. 2015, 274, 1-10. [CrossRef]

10. Jayswal, A. Optimality and duality for nondifferentiable minimax fractional programming with generalized convexity. Appl. Math. 2011. [CrossRef]

11. Dubey, R.; Mishra, L.N.; Mishra, V.N. Duality relations for a class of a multiobjective fractional programming problem involving support functions. Am. J. Oper. Res. 2018, 08, 294-311.

12. Dubey, R.; Mishra, V.N. Symmetric duality results for a nondifferentiable multiobjective programming problem with support function under strongly assumptions. RAIRO-Oper. Res. 2019, 53, 539-558. [CrossRef]

13. Dubey, R.; Mishra, L.N.; Ali, R. Special class of second-order non-differentiable symmetric duality problems with $\left(G, \alpha_{f}\right)$-pseudobonvexity assumptions. Mathematics 2019, 7, 763. [CrossRef]

14. Dubey, R.; Mishra, L.N. Nondifferentiable multiobjective higher-order duality relations for unified type dual models under type-I functions. Adv. Stud. Contemp. Math. 2019, 29, 373-382.

15. Stancu-Minasian, I.M. A sixth bibiliography of fractional programming. Optimization 2006, 55, 405-428. [CrossRef]

16. Stancu-Minasian, I.M. A seven bibiliography of fractional programming. Adv. Model. Optim. 2013, 15, 309-386.

17. Liang, Z.A.; Huang, H.X.; Pardalos, P.M. Optimaity conditions and duality for a class of nonlinear fractional programming problems. J. Optim. Theory Appl. 2002, 110, 611-619. [CrossRef] 
18. Hachimi, M.; Aghezzaf, B. Sufficiency and duality in differentiable multiobjective programming involving generalized type I functions. J. Math. Anal. Appl. 2004, 296, 382-392. [CrossRef]

19. Jayswal, A.; Kummari, K. Second-order duality for nondifferentiable minimax programming problems involving generalized $\alpha$-type-I functions. Int. J. Math. Oper. Res. 2014, 6, 393-406. [CrossRef]

(C) 2019 by the authors. Licensee MDPI, Basel, Switzerland. This article is an open access article distributed under the terms and conditions of the Creative Commons Attribution (CC BY) license (http:/ / creativecommons.org/licenses/by/4.0/). 